\title{
Cerebral autoregulation: Making sense of the nonsensical
}

White matter injury is the most common brain injury in neonates with congenital heart disease ${ }^{1,2}$ because of their vulnerable, immature oligodendrocytes located in watershed areas., ${ }^{3,4}$ Cerebral autoregulation constrains cerebral blood flow (CBF) during fluctuations in arterial blood pressure (ABP). Impaired cerebral autoregulation, commonly seen during hypotension, increases vulnerability to both hypoperfusion and hyperperfusion injuries in the brain. It is therefore intuitively likely but unproven that measurement and optimization of cerebral autoregulation could be used to confer neuroprotection when there is risk of ischemic injury.

In this issue of the Journal, Votava-Smith and colleagues ${ }^{5}$ report their study of cerebral autoregulation in 24 neonates with congenital heart disease by determining coherence between ABP and cerebral oximetry from near-infrared spectroscopy. They concluded that neonates with severe forms of congenital heart disease show periodic impaired cerebral autoregulation, even when normotensive.

Every imaginable proxy of $\mathrm{CBF}$ has been used to measure cerebral autoregulation, including transcranial Doppler ultrasonography, cerebral oximetry, brain tissue oxygen tension, and intracranial pressure. Each of these suggested proxies has been quantitatively related to ABP both by time-domain metrics (analyzing signal associations in time with correlation) and by frequency-domain metrics (deconstructing the signal into components and applying cross-correlation functions, such as coherence and phase).

Complex, chaotic biologic signals such as ABP or cerebral oximetry can be expressed as a summation of sine wave components of different frequencies and amplitudes, which is known as a Fourier transform. When 2 signals share wave components at a given frequency with a constant phase relationship, they are coherent at that frequency. The use of coherence as a metric of autoregulation follows straightforward logic: Autoregulation is a mechanism that constrains CBF fluctuations, so CBF fluctuations coherent with ABP fluctuations are indicative of impaired autoregulation. This assertion is not

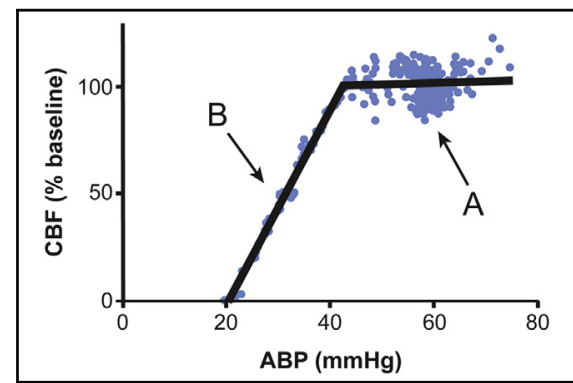

Intact $(A)$ and impaired $(B)$ cerebral autoregulation.

Central Message

Optimization of cerebral autoregulation may allow better neuroprotection of neonates with congenital heart disease; however, there is still no consensus regarding how best to measure autoregulation.

See Article page 1038.

universally accepted, however, because high coherence does not specify a phase relationship. That is, two waves can be $180^{\circ}$ phase shifted (inverted or negatively correlated) and have high coherence, or they can be $0^{\circ}$ phase shifted (positively correlated) and again have high coherence.

Animal data suggest that negative correlation (high coherence with phase shift) between ABP and CBF represents a state of pressure reactivity (ie, intact autoregulation), rather than impaired autoregulation (Figure 1). ${ }^{6-8}$ On the basis of these studies, correlation and phase shift may be better suited to the analysis of autoregulation than is coherence, the method used in this study. ${ }^{9}$

There are competing models of cerebral autoregulation in the literature. According to one, autoregulation acts as a clamp on $\mathrm{CBF}$, such that $\mathrm{ABP}$ coherence with $\mathrm{CBF}$ discriminates intact from impaired autoregulation. According to the other model, autoregulation causes CBF to fluctuate around a set value, such that dynamic vascular resistance causes a phase shift between $\mathrm{ABP}$ and CBF, which thus remain coherent even when autoregulation is intact.

Regardless of the chosen model, any clinical tool proposed to measure autoregulation will face a burden of proof that includes the following: (1) validation against a criterion standard (likely determined in animal models), (2) reproducible prospective evidence that the metric is associated with outcome, and (3) evidence that the metric can be acted on to optimize autoregulation and minimize morbidity. Pilot studies such as this study of Votava-Smith and colleagues ${ }^{5}$ are a first step. 


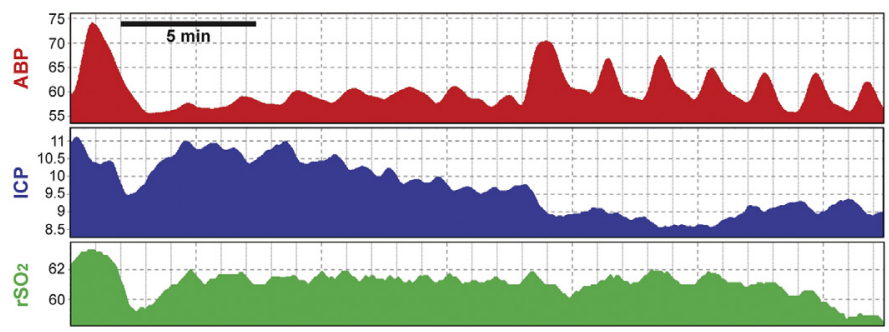

A

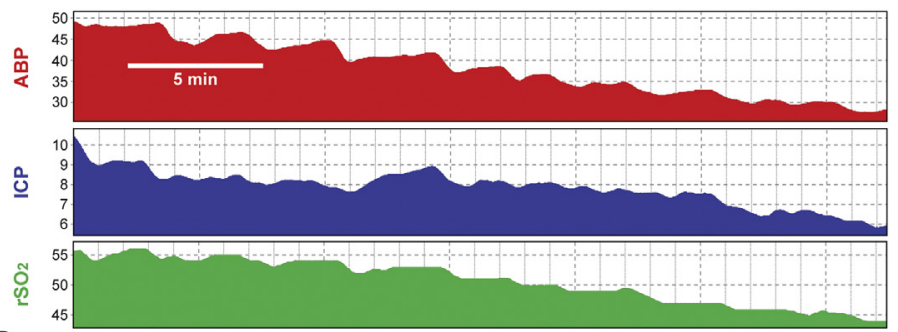

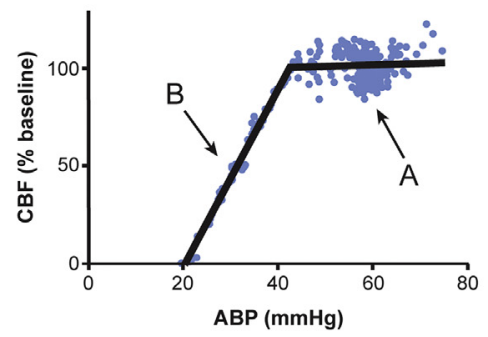

C

\begin{tabular}{|c|c|c|c|c|}
\hline & \multicolumn{2}{|c|}{ Above LLA (A) } & \multicolumn{2}{|c|}{ Below LLA (B) } \\
\hline & Coh. & Phase & Coh. & Phase \\
\hline 10 & 0.71 & $165^{\circ}$ & 0.62 & $34^{\circ}$ \\
\hline $\mathrm{5O}_{2}$ & 0.61 & $150^{\circ}$ & 0.65 & $1^{\circ}$ \\
\hline
\end{tabular}

FIGURE 1. Quantification of cerebral autoregulation from spontaneous blood pressure oscillations in a piglet with hemorrhagic hypotension induced for 4 hours. A, This graph displays changes in arterial blood pressure $(\mathrm{ABP})$, intracranial pressure $(\mathrm{ICP})$, and cerebral oximetry $\left(r \mathrm{So}_{2}\right)$ during $30 \mathrm{minutes}$ of normotension above the lower limit of autoregulation (intact cerebral autoregulation). Slow arterial blood pressure waves (changes across 2-5 minutes) are transmitted to the intracranial pressure and oximetry signals but with an inverted phase (negatively correlated). B, This graph demonstrates the same measurements during 30 minutes of hypotension, below the lower limit of autoregulation (impaired cerebral autoregulation). The slow arterial blood pressure waves are now transmitted without a phase shift (positively correlated). C, A criterion standard static autoregulation plot shows the cerebral blood flow $(C B F)$ from the entire experiment, with arrows showing the position of intact autoregulation recording $(A)$ and the impaired autoregulation recording $(B)$. At steady state, cerebral blood flow is independent of arterial blood pressure above the lower limit of autoregulation and is correlated with arterial blood pressure below the lower limit of autoregulation. D, The table shows coherence $(\mathrm{Coh}$.) and phase shift between arterial blood pressure and the output signals intracranial pressure and cerebral oximetry for panels (A) and (B). During both intact and impaired autoregulation, all signals are coherent. Above the lower limit of autoregulation $(L L A)$, however, there is a phase shift between the arterial blood pressure and output signals. Below the lower limit of autoregulation, there is minimal phase shift between the arterial blood pressure and the output signals. Coherence was measured from the 30-minute segments shown, with a Hamming window that had 7 segments with $40 \%$ overlap in a frequency range of $0.003 \mathrm{~Hz}$ to $0.02 \mathrm{~Hz}$. Phase was calculated with the same parameters.

\section{References}

1. Beca J, Gunn JK, Coleman L, Hope A, Reed PW, Hunt RW, et al. New white matter brain injury after infant heart surgery is associated with diagnostic group and the use of circulatory arrest. Circulation. 2013;127:971-9.

2. Mahle WT, Tavani F, Zimmerman RA, Nicolson SC, Galli KK, Gaynor JW, et al. An MRI study of neurological injury before and after congenital heart surgery. Circulation. 2002;106(12 Suppl 1):I109-14.

3. Volpe JJ. Neurobiology of periventricular leukomalacia in the premature infant. Pediatr Res. 2001;50:553-62.

4. Andropoulos DB, Hunter JV, Nelson DP, Stayer SA, Stark AR, McKenzie ED, et al. Brain immaturity is associated with brain injury before and after neonatal cardiac surgery with high-flow bypass and cerebral oxygenation monitoring. J Thorac Cardiovasc Surg. 2010;139:543-56.

5. Votava-Smith JK, Statile CJ, Taylor MD, King EC, Pratt JM, Nelson DP, et al. Impaired cerebral autoregulation in preoperative newborn infants with congenital heart disease. J Thorac Cardiovasc Surg. 2017:154: $1038-44$.

6. Brady KM, Lee JK, Kibler KK, Easley RB, Koehler RC, Shaffner DH. Continuous measurement of autoregulation by spontaneous fluctuations in cerebral perfusion pressure: comparison of 3 methods. Stroke. 2008;39:2531-7.

7. Brady KM, Lee JK, Kibler KK, Smielewski P, Czosnyka M, Easley RB, et al. Continuous time-domain analysis of cerebrovascular autoregulation using near-infrared spectroscopy. Stroke. 2007;38:2818-25.

8. Lee JK, Kibler KK, Benni PB, Easley RB, Czosnyka M, Smielewski P, et al. Cerebrovascular reactivity measured by near-infrared spectroscopy. Stroke. 2009;40:1820-6.

9. Liu X, Czosnyka M, Donnelly J, Budohoski KP, Varsos GV, Nasr N, et al. Comparison of frequency and time domain methods of assessment of cerebral autoregulation in traumatic brain injury. J Cereb Blood Flow Metab. 2015;35: 248-56. 